\title{
Safe exclusion of pulmonary embolism using the Wells rule and qualitative D-dimer testing in primary care: prospective cohort study
}

The authors of this Research paper would like to point out that a calculation error resulted in an inaccuracy in the figure, which shows the flow of participants through the study (BMJ

2012;345:e6564, doi:10.1136/bmj.e6564). For patients with a Wells score $\leq 4(n=422)$, but a positive point of care D-dimer $(\mathrm{n}=150)$, the total number of venous thromboembolic events should be 17 (not 25, as published).

Cite this as: BMJ 2012;345:e7074

๑ BMJ Publishing Group Ltd 2012 